\title{
El consentimiento informado en Fisioterapia. Estado actual y opinión de los profesionales de Fisioterapia en Castilla y León
}

\author{
Informed Consent in Physiotherapy. Current Status and \\ Professional Opinion of Phyfical Therapy in Castiila y León \\ Mercedes Martínez León \\ Profesora Contratado Doctor de Medicina Legal y Forense. \\ Universidad de Valladolid \\ $M^{\mathbf{a}}$ Teresa Mingo Gómez \\ Profesora del Departamento de Cirugía, Oftalmología, ORL y Fisioterapia. Escuela \\ Universitaria de Fisioterapia. Universidad de Valladolid. Soria. \\ Francisco José Navas Cámara \\ Profesor del Departamento de Bioquímica y Biología Molecular y Fisiología. \\ Escuela Universitaria de Fisioterapia. Universidad de Valladolid. Soria.

\section{Lucía Luisa Pérez-Gallardo} \\ Profesora del Departamento de Bioquímica y Biología Molecular y Fisiología. \\ Escuela Universitaria de Fisioterapia. Universidad de Valladolid. Soria. \\ Isabel Antonina Bayona Marzo \\ Profesora del Departamento de Anatomía Patológica, Microbiología, Medicina \\ Preventiva y Salud Pública, Medicina Legal y Forense. Escuela Universitaria de \\ Fisioterapia. Universidad de Valladolid. Soria.
}

Fecha de recepción del artículo septiembre 2011

Fecha de aceptación del artículo Octubre de 2011

\section{Resumen}

Introducción: El consentimiento informado en fisioterapia es un derecho del paciente con el que mejorar su satisfacción y la del fisioterapeuta. Su ausencia puede dar lugar a indefensión y en última instancia a problemas judiciales. Con este documento el paciente puede elegir libremente sobre su salud ya que se considera el último paso ético y legal para comenzar su tratamiento. El objetivo de este estudio, es realizar un análisis descriptivo de las opiniones, de los fisioterapeutas responsables de Atención Primaria de cada provincia de la comunidad de Castilla y León, acerca de aspectos relacionados con el consentimiento informado.

Material y métodos: Se ha recabado información mediante un cuestionario realizado a los fisioterapeutas responsables de 
Atención Primaria de las provincias de Castilla y León.

Resultados y Conclusiones: Todos los fisioterapeutas contestaron la encuesta. El 27,3\% de estos profesionales consideró no tener suficiente información sobre el consentimiento informado; sólo un 18,2 \% lo cumplimentaban con sus pacientes. El 81,1\% respondió que ningún otro profesional sanitario de su Centro de trabajo lo realizaba. Puesto que no existe un modelo unificado, el $90,9 \%$ cree necesario desarrollar uno como herramienta de calidad y de protección del profesional y del usuario. La mayor parte de los profesionales conoce lo que es el consentimiento informado en fisioterapia pero no lo utiliza. Sin embargo, si cree necesario la elaboración de un modelo de este documento.

\section{Palabras claves}

Consentimiento informado, Fisioterapia, Ética, Legislación, Calidad.

\section{Summary}

Introduction: The informed consent in physical therapy is a patient right that increases its satisfaction and that of the physical therapist. When it is not performed, defenseless and legal problems could arise. With this document the patient can decide freely about its health since it is considered the last ethical and legal step before beginning treatment. The aim of this study is to carry out a descriptive analysis of the opinions of physical therapists responsible for primary care in each province of «Castilla y Leon» (Spain), about issues related to informed consent.

Material and method: The information was obtained through a questionnaire completed by physical therapists responsible for primary care in each province of "Castilla y Leon" (Spain).

Results and Conclusions: All physical therapists completed the questionnaire. $27.3 \%$ considered not having enough information about informed consent; only $18.2 \%$ performed it with their patients. $81.1 \%$ answered that no other health care professional used the informed consent in their work center. Since there is not a form model unified, $90.9 \%$ believe necessary to elaborate one, as a quality and protection tool for professionals and users.Most physical therapists know what the informed consent is but they do not use it. They think that it is necessary to elaborate a model form of this document.

\section{Key words}

Informed Consent, Physical therapy, Ethics, Legislation, Quality.

\section{Introducción}

La ley define el consentimiento informado como «la conformidad libre, voluntaria y consciente de un paciente, manifestada en el pleno uso de sus facultades después de recibir la información adecuada, para que tenga lugar una actuación que afecta a su salud» (1). Puede afirmarse que es en el Código de Nuremberg (1948) donde nace el consentimiento informado y se considera indispensable que la persona que va a participar en un experimento acepte volun- 
tariamente, tras conocer los detalles de su colaboración. Pero no es hasta 1973 cuando, en Estados Unidos, se redacta la primera Carta de Derechos de los Pacientes, donde se recogen sus derechos que darán pie a las primeras demandas y resoluciones judiciales. A partir de ahí, se comprende y se implanta la necesidad de informar al paciente, circunstancia que se recoge en sendos documentos, el Documento de la Asamblea Parlamentaria del Consejo de Europa (1976) y en el Documento AMA (1981) (2).

En nuestro país el derecho a la salud se reconoce en la constitución (3) (1978) y es en la Ley General de Sanidad (4) (1986) donde comienza a transformarse el modelo de las relaciones en la sanidad. En el Convenio de Derechos Humanos y Biomedicina del Consejo de Europa (5) (Convenio de Oviedo1997) aparecen reflejados los derechos del paciente a ser informado, entendiendo que se deben proteger los derechos humanos y la dignidad de los mismos. Si bien es en la Ley 41/2002, de 14 de noviembre, donde se regula detalladamente el derecho a la autonomía del paciente incidiendo en sus derechos y obligaciones en materia de información (1) (art.6,12 y 13) y documentación clínica (art. 4.1 y 8.2).

Ello supone la actualización en el ámbito jurídico y ético de la información que se otorga al paciente, es decir, es una parte de la lex artis. Esta Ley (1) en su artículo 2.6 desarrolla la obligación de todo profesional sanitario, por ende los fisioterapeutas, a intervenir en la actividad asistencial del paciente mediante información y realización de los documentos clínicos pertinentes.

Las comunidades autónomas también han tratado este tema, por ejemplo Cataluña con la aprobación de la Ley 21/2000 sobre los derechos de información concerniente a la salud y la autonomía del paciente, y la documentación clínica (6) o Castilla y León en la Ley 8/2003 sobre Derechos y Deberes de las personas en relación con la salud (7) e incluso han elaborado guías de consentimiento informado (8).

Las personas tras recibir la información deben aprender a tomar decisiones participando de su propia salud, ejerciendo así el derecho de libertad. Este enfoque del concepto libertad se solapa con el principio de autonomía, entendida como la capacidad de conocer por nosotros mismos qué debemos hacer (9), teniendo ambos como objetivo la dignidad de la persona (10). Por ello, el consentimiento informado debe entenderse como un canal de comunicación entre el paciente y el fisioterapeuta y viceversa.

La Ley (1) pauta que la información puede ser transmitida de forma oral o escrita, si bien, debe tener como objetivo la comprensión lingüística del proceso de comunicación por ambas partes. Es frecuente encontrarse con reclamaciones en las unidades de atención al paciente cuya queja es la falta o fallo de la comunicación interpersonal entre los pacientes y los profesionales (11). El consentimiento informado como documento escrito representa el único soporte capaz de demostrar que el paciente ha recibido la información (12).

En la Ley General de Sanidad, Código Deontológico de Fisioterapia, Ley Reguladora de la Autonomía del Paciente se puede observar las referencias que se hacen del derecho de información sanitaria y al consentimiento informado. Así en el artículo 10.5 de la Ley General de Sanidad se puede entender que se debe realizar una explicación por parte del sanitario al usuario comprensible para él, sus familiares o representantes, además de ser una información completa, continuada, verbal o escrita, sobre el proceso que debe incluir el diagnóstico, pronóstico, y posibilidades de tratamiento.

El consentimiento informado que debe realizar el fisioterapeuta como profesional sanitario, teniendo en cuenta sus competencias profesionales, será habi- 
tualmente verbal, enmarcado como un proceso de comunicación continua. Sin embargo, utilizará un documento escrito en el supuesto que se deba aplicar a un paciente algún procedimiento terapéutico invasor o que suponga un riesgo o pueda repercutir de forma negativa a su salud (artic.8.2) (1).

Este registro no busca únicamente tener un documento firmado, sino tener la confirmación de que el paciente ha ejercido su derecho a la información (13), (14).

La jurisprudencia de la Sala Primera del Tribunal Supremo ha catalogado el consentimiento informado como «un derecho humano fundamental, un derecho a la libertad personal, a decidir por sí mismo en lo atinente a la propia persona y a la propia vida y consecuencia de la auto disposición sobre el propio cuerpo».

Y entiende, además, que es «consecuencia necesaria o explicitación de los clásicos derechos a la vida, a la integridad física y a la libertad de conciencia» (Sentencias de 12 de enero de 2001 y 11 de mayo de 2001).

Esta declaración, con la sana intención de resaltar la importancia de este derecho, necesita ser matizada a la hora de llevar a cabo interpretaciones sobre el alcance y contenido de este derecho del paciente.

En primer lugar, la calificación del consentimiento informado como derecho humano fundamental, puede inducir a error, al no quedar claro si con ello se pretende otorgarle la misma categoría que los llamados «derechos fundamentales y libertades públicas» en la Constitución Española, que estarían sujetos a la regulación por Ley Orgánica y garantizados con una tutela específica por los tribunales ordinarios y, en última instancia, a través del recurso de amparo ante el Tribunal Constitucional.

No resulta extraño, por tanto, que algún comentarista haya manifestado que la utilización de la expresión «derecho humano fundamental», respecto al consentimiento informado, por parte del Tribunal Supremo, pueda ser desconcertante, propugnando la conexión del consentimiento informado con los llamados, en el Derecho Privado, «derechos de la personalidad», que sirve para contraponerlos a los derechos patrimoniales y que conlleva una serie de consecuencias en cuanto a su indisponibilidad y condicionamiento respecto de su ejercicio por representación, que se ajustan correctamente con la regulación legal del consentimiento informado.

En sí mismo considerado, el derecho al consentimiento informado por parte del paciente consiste en el otorgamiento por el ordenamiento jurídico de un ámbito de autonomía a la persona para decidir por sí misma acerca de su vida e integridad física, afectada por una enfermedad, con relación al sometimiento por parte de la misma a un tratamiento o actuación de un profesional sanitario.

La jurisprudencia de la Sala de lo Civil del Tribunal Supremo, cuando se refiere al derecho a la información del paciente, necesario con carácter previo para la prestación del consentimiento, lo fundamenta «en la misma Constitución Española, en la exaltación de la dignidad de la persona que se consagra en su artículo 10.1, pero sobre todo, en la libertad, de que se ocupa el artículo 1.1 reconociendo la autonomía del individuo para elegir entre las diversas opciones vitales que se presenten de acuerdo con sus propios intereses y preferencias» (15). 


\section{Material y métodos}

Tras realizar la revisión bibliográfica de la situación actual del consentimiento informado en fisioterapia, se ha cumplimentado un cuestionario (tabla 1) individualizado, durante los meses de mayo y junio de 2011. El procedimiento fue llevado a cabo por un único entrevistador a todos los responsables fisioterapeutas de Atención Primaria $(n=11)$ de las provincias de Castilla y León (dos en Valladolid, dos en León y uno en cada una de las restantes provincias). El cuestionario tenía la intención de recabar información sobre el conocimiento, aplicación y ejecución de dicho documento por parte de los fisioterapeutas a través de un muestreo simple. Dicho cuestionario será ampliado con más variables en un futuro, ya que es el inicio de una línea de investigación, en la que posteriormente se profundizará en su análisis.

\begin{tabular}{|c|c|c|c|}
\hline \multicolumn{4}{|c|}{ Preguntas } \\
\hline 1 & ¿Has oído hablar del consentimiento informado en fisioterapia? & sí & NO \\
\hline 2 & $\begin{array}{l}\text { ¿Cumplimentas el documento de consentimiento informado } \\
\text { en fisioterapia con tus pacientes? }\end{array}$ & sí & NO \\
\hline 3 & $\begin{array}{l}\text { ¿Algún otro profesional de tu Centro de trabajo lo cumplimenta? } \\
\text { En caso afirmativo ¿quién? }\end{array}$ & sí & NO \\
\hline 4 & $\begin{array}{l}\text { ¿Crees necesario un modelo único para todos los fisioterapeutas } \\
\text { asistenciales? }\end{array}$ & sí & NO \\
\hline 5 & $\begin{array}{l}\text { A tu parecer, ¿quién crees que debería encargarse de realizarlo? } \\
\text { Colegios profesionales } \\
\text { Empresa } \\
\text { Profesionales } \\
\text { Multidisciplinar } \\
\text { Otros }\end{array}$ & & \\
\hline
\end{tabular}

\section{Resultados y discusión}

El 100\% de los encuestados respondió voluntariamente al cuestionario.

La Ley garantiza el derecho a la información del paciente como derecho universal mediante el consentimiento informado. Es necesario que los profesionales lo conozcan para que pueda ser aplicado ya que sirve para que el paciente ejerza su decisión libremente y sea partícipe en el proceso de su patología.

Como muestra la figura 1, en nuestro estudio, el $27.3 \%$ de los profesionales fisioterapeutas consideró no tener conocimiento sobre el consentimiento informado en fisioterapia. Iglesias Lepine concluyó en su estudio que la mitad de los profesionales sanitarios desconoce lo que es un consentimiento informado, las partes que lo integran, la ley que lo regula y la filosofía con que fue creado (15).

De los $72,7 \%$ que afirmaron conocer este documento en fisioterapia sólo un $18.2 \%$ lo cumplimentaban con sus pacientes, coincidiendo con los resultados observados por Osuna y col. que considera que el consentimiento informado escrito en fisioterapia, es una práctica muy poco extendida (16), (17) y los que lo manejan habitualmente lo hacen como herramienta de protección ante posibles demandas (18).

Cuando se les preguntó si algún otro profesional sanitario de su Centro de trabajo lo realizaba, el $81.1 \%$ afirmaron que ninguno lo cumplimentaba y el resto 
contestó que desconocía si llevaban a cabo esta práctica.

En nuestro estudio el $90.9 \%$ cree necesario realizar un modelo unificado para el colectivo de fisioterapeutas asistenciales. Son escasos los consentimientos informados de Fisioterapia que hemos encontrado (19), (20) aunque ninguno de ellos se ajusta a todos los apartados que contempla la Ley.

En relación a la $5^{\mathrm{a}}$ pregunta, «A tu parecer, ¿quién crees que debería encargarse de realizarlo?», según este colectivo los responsables de su elaboración serían, en primer lugar un equipo multidisciplinar un $54.5 \%$ (profesionales, representantes de la organización, colegios profesionales y un representante sindical).

En similar proporción un $45.5 \%$ creen que deben ser los propios profesionales o los colegios profesionales los encargados de elaborarlo y sólo uno dice que debe ser la propia empresa quien debiera gestionarlo.

Figura 1

Porcentajes de respuesta de las preguntas del cuestionario

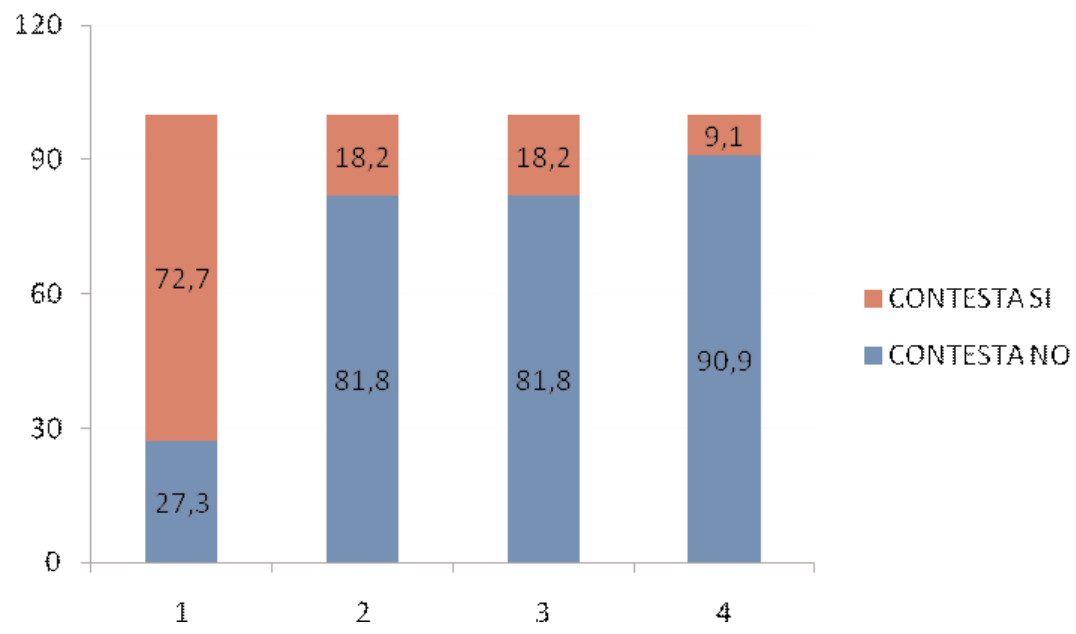

\section{Conclusiones}

La mayor parte de los fisioterapeutas de Atención Primaria conoce lo que es el consentimiento informado en fisioterapia pero no lo utiliza. A la vista de los resultados, parece necesario establecer un documento escrito de consentimiento informado en la práctica profesional de la fisioterapia, como herramienta de calidad y de protección tanto para el usuario como para el profesional.

2 El consentimiento informado no excluye que el fisioterapeuta deba implicarse en la decisión que ha de tomar el paciente libremente ya que posee más información y experiencia clínica que éste. 


\section{Bibliografía}

1. Ley $41 / 2002$, de 14 de noviembre, básica reguladora de la autonomía del paciente y de derechos y obligaciones en materia de información y documentación clínica. (BOE, número 274, de 15-11-2002).

2. SIMÓN P. El consentimiento informado. $1^{\text {a }}$ ed. Madrid: Triacastela, 2000.

3. Constitución Española. (consultada el 15 de mayo de 2011). Disponible en: http://www.la-moncloa.es/NR/rdonlyres/79FF2885-8DFA-4348-845004610A9267F0/0/constitucion_ES.pdf.

4. Ley Orgánica 3/1986, de 14 de abril, de Medidas Especiales en Materia de Salud Pública. (BOE, número 102, de 29-4-1986).

5. Instrumento de Ratificación del Convenio para la protección de los derechos humanos y la dignidad del ser humano con respecto a las aplicaciones de la Biología y la Medicina (Convenio relativo a los derechos humanos y la biomedicina), hecho en Oviedo el 4 de abril de 1997.(BOE, número 251, de 20-10-1999).

6. Llei $21 / 2000$, de 29 de desembre, sobre els drets d'informació concernent la salut i l'autonomia del pacient, i la documentació clínica. (DOGC número 3303, de 11-1-2001).

7. Ley 8/2003 de Ordenación del Sistema Sanitario de Castilla y León, de 8 de abril, sobre Derechos y Deberes de las personas en relación con la salud. (BOE, número 103, de 30-4-2003).

8. Guía de Consentimiento Informado. Junta de Castilla y León. Consejería de Sanidad, 2004.

9. CAmPS V. La voluntad de vivir. Barcelona: Ariel, 2005.

10. MorelLI MG. Bioética, derechos humanos, discriminación y experimentación. Cuadernos de Bioética.2000; 1:134-45.

11. Mirallas Martínez JA: Medicina defensiva y Consentimiento Informado en rehabilitación. Rehabilitación. 2003; 37(2):65-6.

12. Del Brio MA et Al. Reflexiones sobre el Consentimiento Informado. Propuesta para el análisis de los documentos de Consentimiento Informado. Rev. Calidad Asistencial.2006; 21(4):213-9.

13. Pantoja Zarza L. El consentimiento informado: ¿sólo un requisito legal? Rev. Esp. Reumatol. 2004; 31(8):475-8.

14. NavarRo Royo C. Legibilidad de los documentos de consentimiento informado del Hospital de la Paz. Rev. Calidad Asistencial.2002; 17(6):331-6.

15. De Lorenzo Montero R. El Consentimiento Informado como Derecho Humano Fundamental. Publicado en Redacción Médica el Martes 9 de junio de 2009. Número 1022. Año V.

16. IGLESIAS LEPINE ML. Consentimiento informado: opiniones del personal sanitario de un hospital universitario. Rev. Clin. Esp. 2007; 207(10):483-8.

17. Osuna Pérea MC, López Ruiz mC, Díaz Fernández A, Sánchez Alcalá M. Consentimiento informado, Fisioterapia y sanidad pública: Un estudio descriptivo transversal multicéntrico. Cuestiones de Fisioterapia.2010; 39(2):95-103. 
18. Valbuena Moya SO. El Consentimiento informado en la actividad asistencial fisioterápica. [Tesis doctoral]. Murcia: Facultad de Medicina; 2000.

19. BROGGI MA. El peligro de la práctica defensiva en la aplicación del Cl escrito. Rev. Calidad Asistencial.1999; 14:122-7.

20. Colegio de Fisioterapeutas de Baleares. (consultada el 15 de mayo de 2011). Disponible en:

http://www.colfisiobalear.org/descargas/descargas_oficiales/12_consentimiento_informa do.pdf.

21. Colegio de Fisioterapeutas de Castilla y León. (consultada el 15 de mayo de 2011). Disponible en: http://www.cpfcyl.com/downloads.htm.

Correspondencia

Mercedes Martínez León c/ María de Molina, 6, $4^{\circ}$ Izda 47001 Valladolid

Teléfono: +34983423065

Facultad: Mañanas

Teléfono: +34 606107021

legal@med.uva.es 